\title{
Effects of the Constituents of Gardenia Fructus on Prostaglandin and NO Production
}

\author{
Hyun Lıм¹, Kwang-Rock Park ${ }^{2}$, Dong-Ung LeE ${ }^{2}$, Yeong Shik Kıм³ and Hyun Pyo Kıм* \\ ${ }^{1}$ College of Pharmacy, Kangwon National University, Chunchon 200-701, Korea \\ ${ }^{2}$ Dept. Biotechnology, Dongguk University, Gyeongju 780-714, Korea \\ ${ }^{2}$ Natural Products Research Institute and College of Pharmacy, Seoul National University, Seoul 110-460, Korea
}

(Received February 11, 2008; Accepted April 3, 2008)

\begin{abstract}
The fruits of Gardenia jasminoides Ellis have been previously reported to possess anti-inflammatory activity. In this study, the constituents including geniposide, geniposidic acid, genipin and crocin were evaluated for their effects on prostaglandin and NO production in an attempt to establish anti-inflammatory cellular mechanisms. Among the constituents tested, only genipin significantly inhibited cyclooxygenase-2-mediated $\mathrm{PGE}_{2}$ and inducible nitric oxide synthase-mediated NO production from lipopolysaccharide-treated RAW 264.7 cells at 10 $-100 \mu \mathrm{M}$. Genipin also inhibited nuclear transcription factor-kB activation. Moreover, genipin showed in vivo antiinflammatory activity on $\lambda$-carrageenan-induced paw edema in mice (10.4-29.9\% inhibition at $20-100 \mathrm{mg} / \mathrm{kg}$, i.p.). All of these results suggest that genipin may contribute to anti-inflammatory activity of the fruits of $G$ jasminoides and an inhibitory action on prostaglandin and NO production is, at least, the part of anti-inflammatory mechanism of genipin.
\end{abstract}

Keywords: Gardenia jasminoides Ellis, genipin, cyclooxygenase, nitric oxide synthase, anti-inflammation

\section{INTRODUCTION}

Among various proinflammatory chemical mediators, prostaglandins (PG) synthesized from arachidonic acid (AA) play an important role in many inflammatory disorders. Cyclooxygenases (COX) are the enzymes responsible for synthesizing PGs. Especially, COX-2 (an inducible isoform of COX) produces high amounts of $P G s$ in inflammatory lesions. In addition, nitric oxide (NO) synthesized from arginine by nitric oxide synthase (NOS) is also involved in some inflammatory disorders. In particular, inducible NOS (iNOS) produces massive amount of NO in certain cell types including macrophages (Gallin and Snyderman, 1999). Thus, it is worthy to evaluate the effects of potential anti-inflammatory agents on prostaglandin and NO production.

In Chinese medicine, the fruits of Gardenia jasminoides Ellis (Rubiaceae) have been used on inflammatory conditions, jaundice, diarrhea, etc (Bae, 2000). Previously, the alcoholic extract of Gardenia fruits and geniposide showed favorable effects on soft tissue injury (Yao et al., 1991). Hydrophobic pigment in Gardenia inhibited IgE production from rat spleen lymphocytes (Kuramoto et al., 1996). In addition, the fruits of Gardenia protected from pancreatitis by reducing blood circulation in rats (Jia et al., 1993). Recently, genipin was found to inhibit iNOS expression leading to reduced production of NO by inhibiting nuclear transcription factor- $\mathrm{KB}$ (NF-kB) pathway (Koo et al., 2004), and it was also reported that genipin and geniposide including the alcoholic extract showed in vivo anti-inflammatory activity on several animal models of acute inflammation (Koo et al., 2006). Although these previous reports may explain some anti-inflammatory property of the fruits of $G$ jasminoides, anti-inflammatory active constituents from the same plant material have not been fully understood. Therefore, in the present study, anti-inflammatory activity of geniposide, geniposidic acid, genipin and crocin successfully isolated from the fruits of $G$ jasminoides was investigated using in vitro and in vivo models to define anti-inflammatory activity further.

\footnotetext{
${ }^{*}$ Corresponding author

Tel: +82-33-250-6915, Fax: +82-33-255-9271

E-mail: hpkim@kangwon.ac.kr
} 


\section{MATERIALS AND METHODS}

\section{Chemicals}

$\mathrm{N}$-[2-cyclohexyloxy-4-nitrophenyl]methane sulfonamide (NS-398) was obtained from Biomol (Plymouth Meeting, PA). 2-Amino-5,6-dihydro-6-methyl-4H-1,3-thiazine hydrochloride (AMT) was purchased from Tocris Cookson Ltd. (UK). 3-(4,5-Dimethylthiazol-2-yl)-2,5-diphenyltetrazolium bromide (MTT) and LPS (Escherichia coli 0127:B8) were purchased from Sigma Chem. (St. Louis, MO). LipofeCAMINE PLUS, DMEM and other cell culture reagents including FBS were products of Gibco BRL (Grand Island, NY). Protein assay kit was purchased from Bio-Rad Lab. (Hercules, CA). Geniposide, geniposidic acid, genipin and crocin (Fig. 1) were isolated from the butanol fraction of the fruits of $G$ jasminoides and their chemical structures were identified according to the previously described (Lee et al., 2005; Kim et al., 2006). Test compounds dissolved in DMSO were diluted with serum-free DMEM into appropriate concentrations. Final concentration of DMSO in the culture medium was adjusted to $0.1 \%(\mathrm{v} / \mathrm{v})$.

\section{RAW 264.7 cell culture and measurement of $\mathrm{NO}$ and $\mathrm{PGE}_{2}$ concentrations}

RAW 264.7 cells obtained from American Type Culture Collection (ATCC, Rockville, MD) were cultured in DMEM supplemented with $10 \%$ FBS and $1 \%$ antibiotics under $5 \% \mathrm{CO}_{2}$ at $37^{\circ} \mathrm{C}$ based on the previously described procedures (Chi et al., 2001). Briefly, cells were plated in 96 -well plates $\left(2 \times 10^{5}\right.$ cells/well). After pre-incubation for $2 \mathrm{~h}$, the test compounds and LPS $(1 \mu \mathrm{g} / \mathrm{ml})$
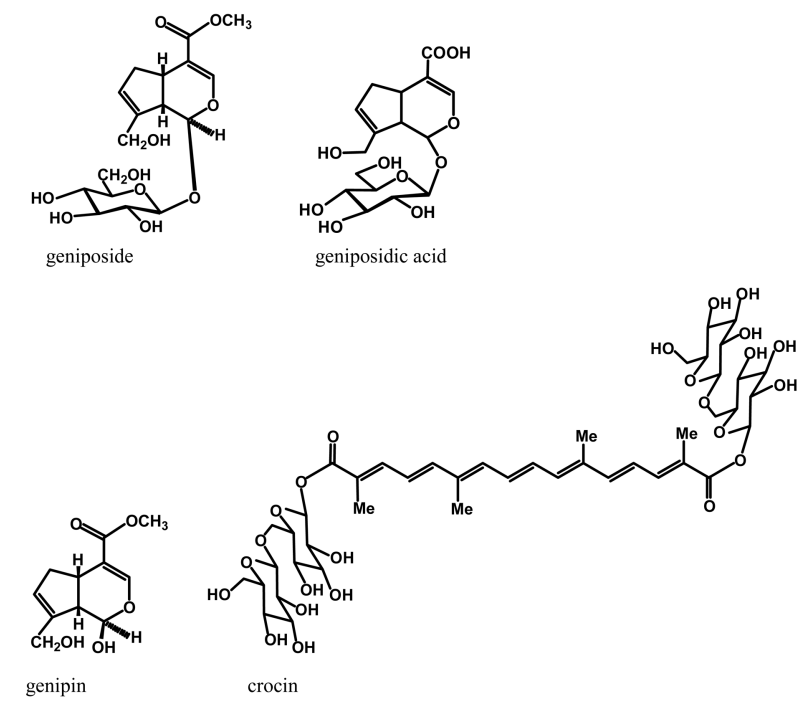

Fig. 1. The chemical structures of the constituents studied. were added and incubated for $24 \mathrm{~h}$. From the media, NO and $\mathrm{PGE}_{2}$ concentrations were measured. For determination of NO concentration, the stable conversion product of $\mathrm{NO}$, nitrite $\left(\mathrm{NO}_{2}{ }^{-}\right)$, was measured using Griess reagent and optical density was checked at $550 \mathrm{~nm}$. $\mathrm{PGE}_{2}$ concentration in the medium was measured using ELISA kit for $\mathrm{PGE}_{2}$ (Cayman Chem. Co.) according to the manufacturer's recommendation. Cell viability was assessed with MTT assay as described previously (Mossman, 1983).

\section{Western blot analysis of iNOS}

For measuring the protein level of iNOS, Western blotting technique was used (Chi et al., 2001). RAW cells were cultured in 6 -well plates $\left(5 \times 10^{6}\right.$ cells/well) in the presence or absence of LPS $(1 \mu \mathrm{g} / \mathrm{ml})$ with/without genipin for $16-20 \mathrm{~h}$. After preparing cell homogenate, the supernatant was obtained by centrifugation at $15,000 \mathrm{~g}$ for $30 \mathrm{~min}$. Using Tris-glycine gel (8\%), electrophoresis was carried out and bands were blotted to PVDF membranes. iNOS antibody (N32030, Transduction Lab.) was incubated and bands were visualized with HRP-linked secondary antibody (Cell signaling, Denver, MA) and chemiluminescent reagent (Amersham, UK).

\section{Electrophoretic mobility shift assay (EMSA)}

RAW cells were treated with/without LPS and genipin for $3 \mathrm{~h}$. To prepare nuclear fractions, the cells were washed with PBS, harvested and resuspended in $400 \mu \mathrm{l}$ of buffer A (10 mM HEPES, $10 \mathrm{mM} \mathrm{KCl}, 0.1 \mathrm{mM}$ EDTA, $1 \mathrm{mM}$ DTT, $0.5 \mathrm{mM}$ PMSF, pH 7.9) for $15 \mathrm{~min}$ on ice. After $10 \%$ NP-40 (25 $\mu \mathrm{l})$ was added, the tubes were vortexed vigorously for $10 \mathrm{sec}$. The nuclei were collected by centrifugation at 5,000 rpm for $3 \mathrm{~min}$ and the supernatant was saved as the cytosolic fraction. The nuclei were lysed in buffer B (20 mM HEPES, $0.4 \mathrm{M} \mathrm{NaCl}, 1 \mathrm{mM}$ EDTA, 1 mM DTT, 1 mM PMSF, pH 7.9). NF-kB consensus oligonucleotide (Promega) was phosphorylated by $\mathrm{T} 4$ polynucleotide kinase (10 units) with $10 \mu \mathrm{Ci}$ of $\left[\gamma^{-32} \mathrm{P}\right]$ ATP $(3,000 \mathrm{Ci} / \mathrm{mmol})$ at $37^{\circ} \mathrm{C}$ for $10 \mathrm{~min}$. Unincorporated oligonucleotides were removed by Microspin G-25 column (Amersham, UK). Nuclear extract containing $5 \mu \mathrm{g}$ protein was incubated with ${ }^{32} \mathrm{P}$-labeled NF-KB consensus oligonucleotide in gel shift binding buffer at room temperature for $20 \mathrm{~min}$. The incubation mixture was subjected to electrophoresis on a $4 \%$ polyacrylamide gel in TBE buffer $(0.5 \mathrm{X})$ at $350 \mathrm{~V}$. The gel was dried and exposed to X-ray film overnight at $-70^{\circ} \mathrm{C}$.

\section{$\lambda$-Carrageenan (CGN)-induced paw edema in mice \\ In order to examine in vivo anti-inflammatory activity,}


mouse CGN-induced paw edema assay was used according to the slightly modified procedures of Lichtman et al. (2004) based on the original methods of Winter et al. (1962). Specific-pathogen free male ICR mice were purchased from Orient-Bio (Seoul, Korea) and acclimatized in animal facility with standard lab. chow and water ad libitum at least for 7 days prior to experiment. Test compounds dissolved in DMSO were administered intraperitoneally to mice $(0.05 \mathrm{ml} /$ mouse). One hour later, $1 \%$ CGN (w/v) dissolved in pyrogen-free sterile saline solution $(0.05 \mathrm{ml} / \mathrm{paw})$ was injected to right hind paw. Five hours later, paw volume was measured using plethysmometer (Ugo Basil, Italy). The paw volume increased from the initial non-treated paw volume was regarded as edema.

\section{Statistical analysis}

Experimental values were represented as arithmetic mean $\pm S D$. Unpaired Student's t-test was used to determine the statistical significance.

\section{RESULTS AND DISCUSSION}

It is well known that RAW 264.7 cells, a mouse macrophage-like cell line, induce COX-2/iNOS which produce high amounts of PGs and NO by LPS treatment (Chi et al., 2001). When LPS $(1 \mu \mathrm{g} / \mathrm{ml})$ was added and the cells were incubated for $24 \mathrm{~h}, \mathrm{PGE}_{2}$ and NO production from the culture media increased to $89.3 \pm 4.4 \mathrm{nM}$ and $38.1 \pm 0.2 \mu \mathrm{M}$ from the basal levels of $2.4 \pm 0.0 \mathrm{nM}$ and 0.6 $\pm 0.0 \mu \mathrm{M}$, respectively $(n=3)$. When the compounds were simultaneously added with LPS and the inhibitory activities were examined, only genipin significantly inhibited COX-2-mediated $\mathrm{PGE}_{2}$ and iNOS-mediated NO production from LPS-treated RAW 264.7 cells at $10-100 \mu \mathrm{M}$ (Fig. 2). Especially, genipin strongly inhibited NO production $\left(\mathrm{IC}_{50}\right.$ for $\mathrm{PGE}_{2}$ production $\left.=58.0 \mu \mathrm{M}\right)$, being less active on $\mathrm{PGE}_{2}$ production. Genipin showed $40.7 \%$ inhibition against $\mathrm{PGE}_{2}$ production at $100 \mu \mathrm{M}$, while other compounds tested did not show a significant inhibition at the concentrations up to $100 \mu \mathrm{M}$. Under the same experimental conditions, the reference compounds, NS-398 (selective COX-2 inhibitor) and AMT (iNOS inhibitor) showed $99.4 \%$ and $95.6 \%$ inhibition at 0.1 and $1.0 \mu \mathrm{M}$, respectively, against COX-2- and iNOS-mediated $\mathrm{PGE}_{2}$ and NO production as expected (Fig. 2). At the concentrations examined, all of the test compounds did not show cytotoxic effects on RAW cells revealed by MTT assay (data not shown). The control experiment without LPS treatment revealed that geniposide, geniposidic acid, genipin (a)

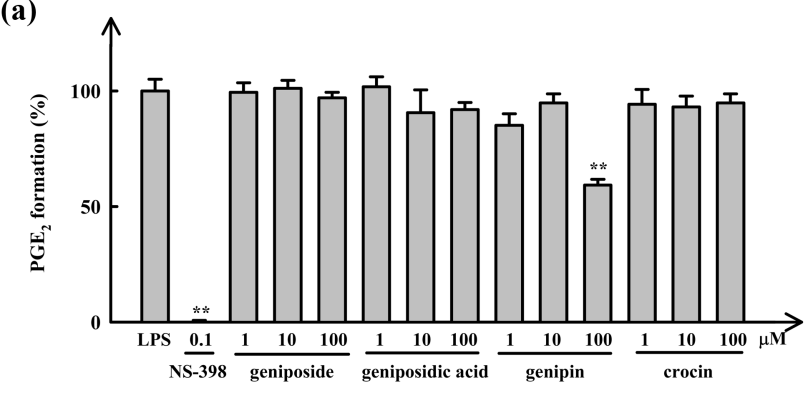

(b)

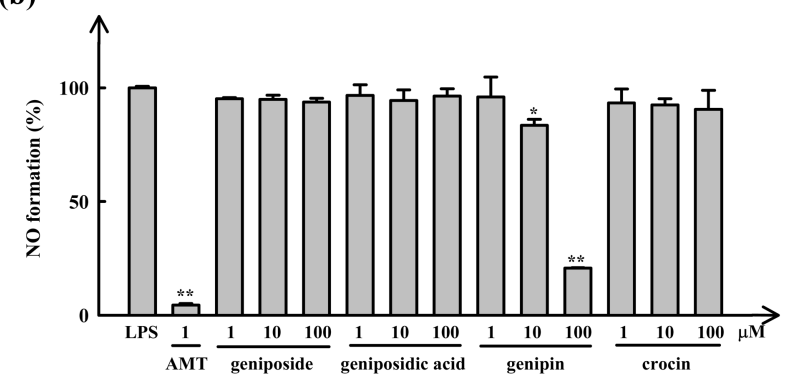

Fig. 2. Effect of the constituents from $G$ jasminoides on COX2-mediated $\mathrm{PGE}_{2}$ and iNOS-mediated NO production from LPS-treated RAW 264.7 cells. (a) Effect on COX-2-mediated $\mathrm{PGE}_{2}$ production. (b) Effect on iNOS-mediated NO production. Data points and bars represent arithmetic mean $\pm S D$ ( $=3)$. ${ }^{*}: P<0.05,{ }^{* *}: P<0.01$, Significantly different from the LPStreated control group.

and crocin did not significantly affect $\mathrm{PGE}_{2}$ and $\mathrm{NO}$ concentration in RAW 264.7 cells at $100 \mu \mathrm{M}$ (data not shown).

In order to elucidate the cellular mechanism of NO inhibition, iNOS expression level was examined using Western blotting analysis. But genipin did not down-regulate iNOS (Fig. 3a). On the other hand, genipin was demonstrated to inhibit NF-KB activation revealed by EMSA (Fig. 3b). It was previously reported that genipin inhibited iNOS expression from macrophages at $300 \mu \mathrm{M}$ (Koo et al., 2004). In the present investigation, the maximum concentration examined was $100 \mu \mathrm{M}$, since the concentrations higher than $100 \mu \mathrm{M}$ are not likely attained in the body by oral genipin ingestion. Thus, iNOS down-regulating capacity of genipin could not be observed in the present study. Our results clearly demonstrated that genipin inhibited NF-KB activation, without iNOS down-regulation at $100 \mu \mathrm{M}$. The precise reason for this inconsistency is not known at present, but it is speculated that the sensitivity of EMSA using radioactivity is higher than that of Western blotting analysis using antigen-antibody reaction. Therefore, it is suggested that genipin may inhibit NO production at least in part by iNOS down-regulation 
(a)

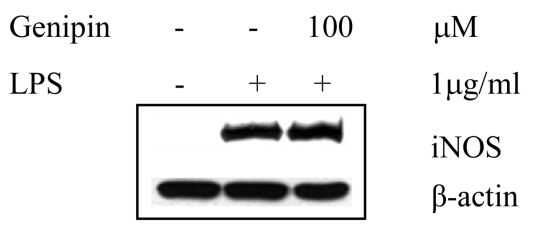

(b)

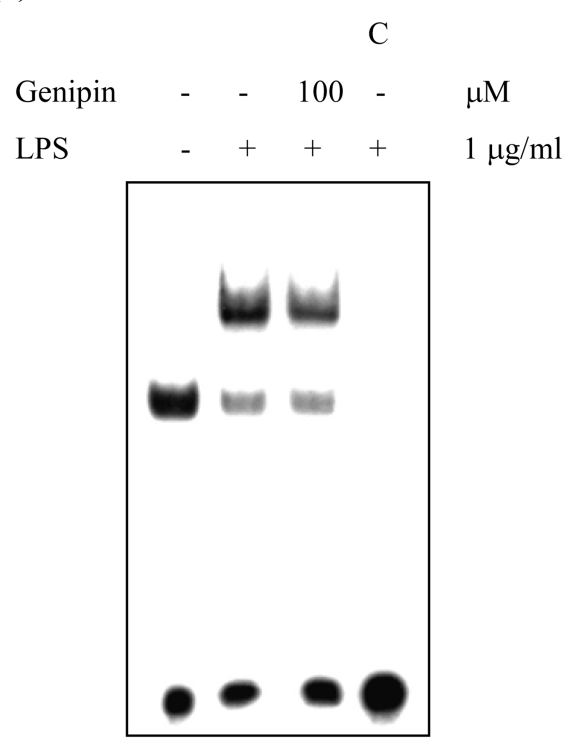

Fig. 3. Western blotting analysis and EMSA. (a) iNOS expression (Western blot), (b) EMSA of NF-KB. Note: Genipin clearly inhibited NF-kB activation, while no effect was observed on iNOS expression at $100 \mu \mathrm{M}$.

via NF-кB inhibition.

In addition, genipin inhibited CGN-induced paw edema in mice by intraperitoneal injection. As shown in Fig. 4, genipin showed $10.4 \%$ and $29.9 \%$ inhibition of paw edema at 20 and $100 \mathrm{mg} / \mathrm{kg}$, respectively. Some of anti-inflammatory activities of genipin were previously described (Koo et al., 2004 and 2006) and NO inhibitory action was repeatedly found in this study. But, the finding of COX-2 inhibitory activity of genipin is new and is important since prostanoids play a critical role in some inflammatory disorders. It is significant to note that genipin derivatives such as geniposide and geniposidic acid did not show anti-inflammatory activity in vitro probably due to inaccessibility to cell inside. The similar results were previously demonstrated that some flavonoid glycosides did not inhibit iNOS-mediated NO production from RAW cells in contrast to the inhibitory action of the respective flavonoid aglycones (Kim et al., 1999).

In conclusion, the present investigation has shown that

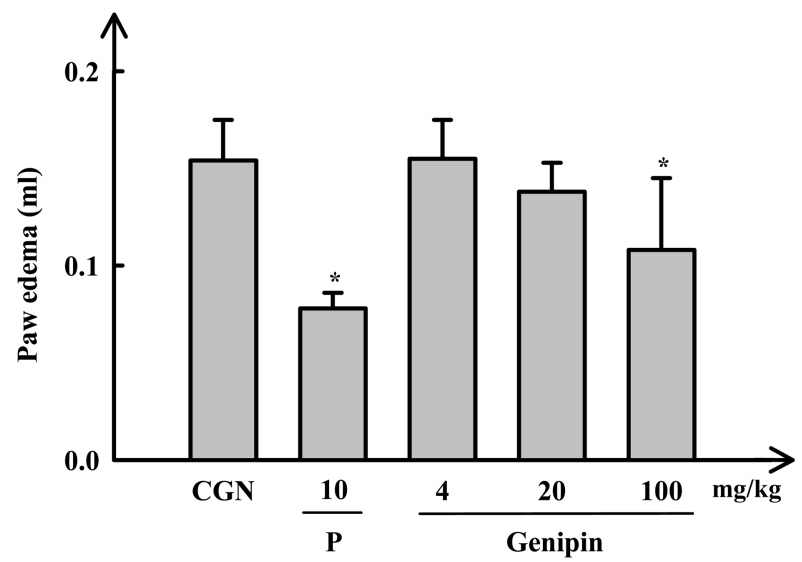

Fig. 4. In vivo anti-inflammatory activity of genipin (CGNinduced mouse paw edema). All compounds were intraperitoneally administered $1 \mathrm{~h}$ prior to CGN injection. $\mathrm{P}$ (prednisolone). Data points and bars represent arithmetic mean $\pm S D$ ( $n$ $=5)$. *: $P<0.05$, Significantly different from the CGN-treated control group.

genipin has anti-inflammatory activity in vitro and in vivo among the constituents tested. Genipin inhibited COX-2mediated $\mathrm{PGE}_{2}$ production and iNOS-mediated NO production. Genipin may reduce prostaglandin and NO concentration in inflammatory lesions, leading to antiinflammatory effect. It is suggested that genipin, among the constituents, may contribute to anti-inflammatory activity of the fruits of $G$ jasminoides.

\section{ACKNOWLEDGMENTS}

This study was financially supported by the research fund of Studies on the Identification of the Efficacy of Biologically Active Components from Oriental Herbal Medicines from Korean Food and Drug Administration (2007) and post-BK21 project from Ministry of Education, Korea.

\section{REFERENCES}

Bae K. 2000. Medicinal plants of Korea, Kyo-Hak Pub. Seoul, pp. 417.

Chi, Y. S., Cheon, B. S. and Kim, H. P. (2001). Effect of wogonin, a plant flavone from Scutellaria radix, on the suppression of cyclooxygenase-2 and the induction of inducible nitric oxide synthase in lipopolysaccharide-treated RAW 264.7 cells. Biochem. Pharmacol. 61, 1195-1203.

Gallin, J. I. and Snyderman, R. (1999). Overview. In Inflammation: Basic principles and clinical correlates $3^{\text {rd }}$ ed. (Gallin, J. I. and Snyderman, R., Eds.), pp. 1-4. Lippincott Williams \& Wilkins, Philadelphia.

Jia, Y. J., Jiang, M. N., Pei, D. K., Ji, X. P. and Wang, J. M. (1993). Effect of Gardenia jasminoides Ellis (GJE) on the 
blood flow of internal organs at the early stage of acute necrotizing hemorrhagic pancreatitis in rats. Chung Kuo Chung Yao Tsa Chih 18, 431-433.

Kim, H. J., Kim, E. J., Seo, S. H., Shin, C. G., Jin, C. and Lee, Y. S. (2006). Vanillic acid glycoside and quinic acid derivatives from Gardeniae Fructus. J. Nat. Prod. 69, 600-603.

Kim, H. K., Cheon, B. S., Kim, Y. H., Kim, S. Y. and Kim, H. P. (1999). Effects of naturally occurring flavonoids on nitric oxide production in the macrophage cell line RAW 264.7 and their structure-activity relationships. Biochem. Pharmacol. 58, 759765.

Koo, H. J., Lim, K. H., Jung, H. J. and Park, E. H. (2006). Antiinflammatory evaluation of gardenia extract, geniposide and genipin. J. Ethnopharmacol. 103, 496-500.

Koo, H. J., Song, Y. S., Kim, H. J., Lee, Y. H., Hong, S. M., Kim, S. J., Kim, B. C., Jin, C., Lim, C. J. and Park, E. H. (2004). Antiinflammatory effects of genipin, an active principle of gardenia. Eur. J. Pharmacol. 495, 201-208.

Kumamoto, Y., Yamada, K., Tsuruta, O. and Suganon, M. (1996). Effect of natural food colorings on immunoglobulin production in vitro by rat spleen lymphocytes. Biosci. Biotechnol.
Biochem. 60, $1712-1713$.

Lee, I. A., Lee, J. H., Baek, N. I. and Kim, D. H. (2005). Antihyperlipidemic effects of crocin isolated from the frutus of Gardenia jasminoides and its metabolite crocetin. Biol. Pharm. Bull. 28, 2106-2110.

Lichtman, A. H., Shelton, C. C., Advani, T. and Cravatt, B. F. (2004). Mice lacking fatty amide hydrolase exhibit a cannabinoid receptor-mediated phenotypic hypoalgesia. Pain 109, 319-327.

Mossman, T. (1983). Rapid colorimetric assay for cellular growth and survival: Application to proliferation and cytotoxic assay. J. Immunol. Methods 65, 55-63.

Winter, C.A., Risley, E.A. and Nuss, G.W. (1962). Carrageenaninduced edema in the hindpaw on the rat as an assay for anti-inflammatory drugs. Proc. Soc. Exp. Biol. Med. 111, 544547.

Yao, O., Zhou, G., Zhu, Y., Pan, Y., Hu, J., Xue, H. and Zhang, Q. (1991). Screening studies on anti-inflammatory function of traditional Chinese herb Gardenia jasminoides Ellis and its possibility in treating soft tissue injuries in animals. Chung Kuo Chung Yao Tsa Chih 16, 489-493 (1991). 